\title{
Usando tecnología móvil para el aprendizaje de la estructura de los elementos Químicos
}

\section{Using mobile technology to learn the structure of chemical elements}

JERONIMO-YEDRA, Rubén†*, ALMEIDA, M. Alejandrina, ARCEO-M. Gerardo y GÓMEZ-R., José L.

Universidad Juárez Autónoma de Tabasco, Av. Universidad s/n, Zona de la Cultura, Col. Magisterial, Villahermosa, Centro, Tabasco, México. C.P. 86040

ID $1^{\text {er }}$ Autor: Rubén, Jeronimo-Yedra / ORC ID: 0000-0003-1617-7444, CVU CONACYT ID: 669977

ID $1^{\text {er }}$ Coautor: M. Alejandrina, Almeida / ORC ID: 0000-0003-1778-8802, CVU CONACYT ID: 669831

ID $2^{\text {do }}$ Coautor: Gerardo, Arceo-M. / ORC ID: 0000-0002-3016-3631, CVU CONACYT ID: 324653

ID $3^{\text {er }}$ Coautor: José L., Gómez-R. / ORC ID: 0000-0001-7102-8797, CVU CONACYT ID: 670757

DOI: $10.35429 /$ JTAE.2019.7.3.8.17

Recibido: 04 de Enero, 2019; Aceptado 10 de Marzo, 2019

\section{Resumen}

En México el uso de la telefonía móvil ha aumento en los últimos años, lo cual da la idea del impacto que tienen en la vida cotidiana, constituyen un paso hacia el aprendizaje en cualquier momento y en cualquier lugar. Es importante crear aplicaciones usando este tipo de tecnología, que sirvan de apoyo al sector educativo. Ésta investigación, que se realizó bajo un enfoque de tipo mixto, usó como herramientas de recolección de datos al cuestionario, la observación y la entrevista, obteniéndose como resultado una aplicación que hizo uso de la tecnología móvil, enfocada para propiciar el aprendizaje de la estructura de las elementos químicos, como apoyo a los alumnos de bachillerato, en la asignatura de Química; esto debido a que los jóvenes depende del formato impreso que contiene el esquema general de todos los símbolos o de la memorización para aprenderse cada uno de los elementos y sus partes. Aunque en la Web existen varias aplicaciones que circulan de manera gratuita sobre la misma temática, algunas están restringidas a equipos de cómputo y otras fueron creadas para teléfonos smartphone que requieren un pago para descargarlas.

Tecnología móvil, Aprendizaje, Elementos químicos

\begin{abstract}
In Mexico the use of mobile telephony has increased in recent years, which gives the idea of the impact they have on daily life, encouraging the user not to be in a predetermined place and are a step towards learning at any time and in any place. It is important to create applications using this type of technology, to support the education sector. This research, which was conducted under a mixed-type approach, used as tools for data collection to the questionnaire, observation and interview, resulting in an application that made use of mobile technology, focused to promote the learning of the structure of the chemical elements, as support to the students of baccalaureate, in the subject of Chemistry; This is because young people depend on the printed format that contains the general scheme of all the symbols or memorization to learn each of the elements and their parts. Although on the Web there are several applications that circulate for free on the same subject, some are restricted to computer equipment and others were created for smartphone phones that require a payment to download them.
\end{abstract}

Mobile technology, Learning, Chemical elements

Citación: JERONIMO-YEDRA, Rubén, ALMEIDA, M. Alejandrina, ARCEO-M. Gerardo y GÓMEZ-R., José L. Usando tecnología móvil para el aprendizaje de la estructura de los elementos Químicos. Revista de Tecnología y Educación. 2019. 3-7: $8-17$

\footnotetext{
* Correspondencia del Autor: (ruben_yedra@yahoo.com.mx)

$\dagger$ Investigador contribuyendo como primer autor.
} 


\section{Introducción}

Las investigaciones sobre los elementos químicos son muy importantes porque con ellas podemos conocer las propiedades de cada elemento y para qué nos sirve. Todo lo que vemos a nuestro alrededor está formado por elementos químicos, como por ejemplo lo que respiramos y comemos; por ello, es importante conocer las propiedades de los elementos para comprender nuestro organismo o para saber cómo manipularlos en la industria alimentaria, metalúrgica, textil, farmacéutica, entre otras muchas más.

Por otro lado, vale mencionar que hoy en día se ha tenido un rápido avance en la evolución y en el uso de la tecnología celular; aparte de la comunicación convencional, la tecnología celular ha brindado la posibilidad de tener acceso a diferentes herramientas como agenda, calculadora, calendario, al igual que aplicaciones, como por ejemplo, de entretenimiento o educativas.

\section{Planteando la Problemática}

Actualmente, en el nivel medio superior en la república Mexicana se imparte la asignatura de Química, y dentro de sus diferentes temas se aborda con mucha frecuencia la información de los elementos químicos de la tabla periódica, de ahí la importancia de conocer a detalle los datos de estos elementos que están contenidas en los formatos impresos. De manera particular en el segundo semestre de estudio en el Colegio de Bachilleres de Tabasco (COBATAB), se imparte la asignatura de Química, donde es importante conocer los elementos químicos de la tabla periódica, ya que su contenido es utilizado en la mayoría de sus temas.

Usualmente el formato impreso es la herramienta más común dentro de este aprendizaje, ya que facilita la ubicación de los elementos utilizando diferentes colores para cada agrupación. Sin embargo, en muchas ocasiones no se tiene el formato a la mano, y recurrir a la memorización no es lo más recomendable ya que no todos pueden retener la información de manera precisa.
Según datos proporcionados en una entrevista realizada a la experta en el tema del COBATAB, plantel 6, del municipio de Cunduacán, Tabasco en México y que además es la profesora que imparte la asignatura en el segundo semestre, menciona que muchos alumnos logran acreditar este tema con baja calificación porque ellos no siempre llevan su formato impreso y a la hora de realizar los ejercicios en clases no tienen donde consultarlos; aunque también ha detectado que se confían de su memoria para aprenderse cada uno de los elementos y confunden sus diversos atributos que éstos poseen, provocando en muchos casos que reprueben la evaluación de este tema.

\section{Objetivo general}

Crear una aplicación de software que sirva para el aprendizaje de la estructura de la tabla periódica mediante tecnología móvil, como apoyo a los alumnos del COBATAB en la asignatura de Química.

\section{Justificación}

La telefonía desarrollada para móviles e Internet, en los últimos años ha manifestado su crecimiento tecnológico, desarrollando o mejorando aplicaciones para los diferentes sectores de la sociedad, convirtiéndose en dos tecnologías de gran aceptación social. En el caso particular de la tecnología móvil se ha enfocado a la comunicación y al entretenimiento masivo, entre otras funciones.

El INEGI, Instituto Nacional de Estadística y Geografía, en cooperación con la Secretaría de Comunicaciones y Transportes (SCT) y el IFT, Instituto Federal de Telecomunicaciones, publicó la Encuesta Nacional sobre Disponibilidad y Uso de Tecnologías de la Información en los Hogares (ENDUTIH) 2018. De acuerdo con los resultados de la ENDUTIH 2018, en la figura 1 se muestra como ocho de cada diez usuarios de teléfono celular disponen de un celular inteligente (Smartphone).

Entre 2017 y 2018, el número total de usuarios que sólo dispusieron de un celular inteligente tuvo un crecimiento de 7.5 puntos porcentuales, es decir, que pasó de 64.7 millones de personas a 69.6 millones. 
Esto significa que, entre los dos años, la diferencia porcentual de usuarios de este tipo de teléfonos fue de $3.6 \%$, pues en 2017 representaban el 80.2 por ciento y en 2018 el 83.8 por ciento, que representan 69.6 millones de usuarios en México (INEGI,2018).
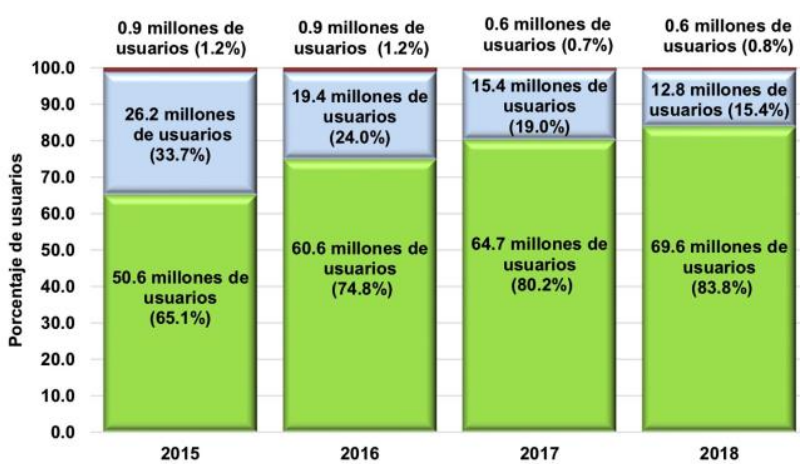

Figura 1 Usuarios de teléfono celular en México Fuente: INEGI, 2018

Muy cierto es que existe la necesidad en el sector laboral y educativo de acceder a los datos en un ambiente móvil por la portabilidad que este representa, así como aplicaciones que permitan obtener la información que se requiera, y al mismo tiempo, que den servicio a los usuarios que se mueven a través de edificios, laboratorios, instituciones educativas, etc. Hoy en día existen diversas aplicaciones que permiten consultar la información de los elementos químicos de la tabla periódica; sin embargo, la mayoría de ellas están desarrolladas para computadoras convencionales y aunque existen algunas para dispositivos móviles, éstas carecen de una interfaz gráfica o utilizan tecnología táctil, de teléfonos caros o se tiene que realizar un pago para poder descargarlas.

A partir de la problemática planteada en la presente investigación, se desarrolló una aplicación que hizo uso de la tecnología móvil, que tuviera como función fundamental ser mediadora y de apoyo específicamente en el proceso de aprendizaje de la estructura general de la tabla periódica. La aplicación de software que se desarrolló en esta investigación está orientado a jóvenes de entre 15 y 16 años, que cursan el segundo semestre de bachillerato, ya que en este semestre cursan la materia de Química, y es aquí donde tienen la necesidad de buscar y conocer la información de los elementos químicos que están contenida en la tabla periódica, ya que la materia así lo requiere porque es parte de su plan de estudio.
Sin embargo, muchos de estos jóvenes tienen la dificultad de no retener la información, ya que el contenido es muy amplio, o simplemente no se tiene a la mano la tabla periódica en el formato impreso convencional cuando se requiere. Esta aplicación tecnológica tiene una estructura organizada, y su interfaz es agradable, fácil de utilizar y manejable, además de esto la aplicación es gratuita.

\section{Marco Teórico}

Sin lugar a dudas, la ciencia no habría podido llegar al lugar preponderante que ocupa en hoy en día dentro del quehacer humano, si los científicos se hubieran conformado con recopilar y acumular datos. Los seres humanos siempre hemos estado tentados a encontrar una explicación a la complejidad de la materia que nos rodea. Al principio se pensaba que los elementos de toda materia se resumían al agua, tierra, fuego y aire. Sin embargo, al cabo del tiempo y gracias a la mejora de las técnicas de experimentación física y química, nos dimos cuenta de que la materia es en realidad más compleja de lo que parece.

En el caso de la Química, hubiese sido muy desalentador desarrollar una ciencia que tuviera como objeto de conocimiento solamente a uno de los elementos o compuestos químicos conocidos, o a un grupo de ellos. Es así que los primeros intentos de clasificación de los elementos químicos estén basados en sus propiedades físicas más sobresalientes.

Cuando se estudian también los aspectos químicos (menos evidentes), se pueden encontrar correlaciones más fuertes e incluso subclasificaciones. Pero cuando se encuentra una característica primordial (parámetro) para los elementos, de la cual dependen las demás propiedades, entendemos que el avance es realmente fundamental y esencial (Martínez, 2010). De todo éste proceso surge la Tabla Periódica de los Elementos Químicos, que asociamos generalmente al nombre del científico ruso Dimitri Ivanovich Mendeleiev.

La historia de la tabla periódica está vinculada al descubrimiento de los diversos elementos químicos y a la necesidad de ordenarlos de alguna forma. Tras varios intentos, Mendeleiev fue quien logró crear un sistema periódico en base a la masa atómica. 
Los 118 elementos que forman la Tabla Periódica actual se distribuyen en columnas (denominadas "grupo" o "familia") y filas (denominadas "periodos”) y están divididos en tres grandes categorías: Metales, Metaloides y No Metales (Química y Sociedad, 2019).

a. Los Metales son los más abundantes y se subdividen en 6 subgrupos: Alcalinos (columna 1), Alcalinotérreos (columna 2), Metales de transición/ Bloque D (columnas 3 a la 12), Lantánidos (fila 6), Actínidos (fila 7) y Otros Metales (columnas 13 a la 16).

b. Los Metaloides son siete de los elementos de la Tabla, distribuidos entre las columnas 13 y 16 .

c. Los No Metales se subdividen en: Otros No Metales (columnas 14 a la 16), Halógenos (columna 17) y Gases Nobles (columna 18).

La distribución de los elementos en la tabla periódica viene determinada por el número atómico y por su configuración electrónica (número de electrones en su capa más externa). Esta distribución guarda un esquema coherente que facilita la comprensión y ordenación de los elementos en la tabla.

Existen 18 grupos en la tabla y los elementos incluidos en cada uno de los grupos comparten la configuración electrónica, lo que determina sus propiedades físicas y químicas. El periodo en el que se encuentran determina el número de capas de electrones que poseen.

Los elementos químicos se representan en la tabla con un símbolo único, acompañado de un número que especifica el número de protones que contiene su átomo y se denomina "número atómico"; y un "número másico", que se refiere a la suma de protones y de neutrones que existe en el núcleo del átomo en cuestión, a como se muestra en la figura 2.

Los neutrones sirven como una especie de pegamento que ayuda a mantener juntos a los protones. Sin ellos, la carga positiva apartaría a unos de los otros. Cuando un átomo tiene el mismo número atómico que otro, es decir, contiene el mismo número de protones, pero diferente número de neutrones, recibe el nombre de "isótopo".
También existe una peculiaridad en el núcleo de átomos muy pesados, como el uranio, ya que están tan llenos de protones que se repelen entre ellos. Este tipo de átomos pasan por una "desintegración radioactiva", es decir, emiten partículas y energía (Química y Sociedad, 2019).

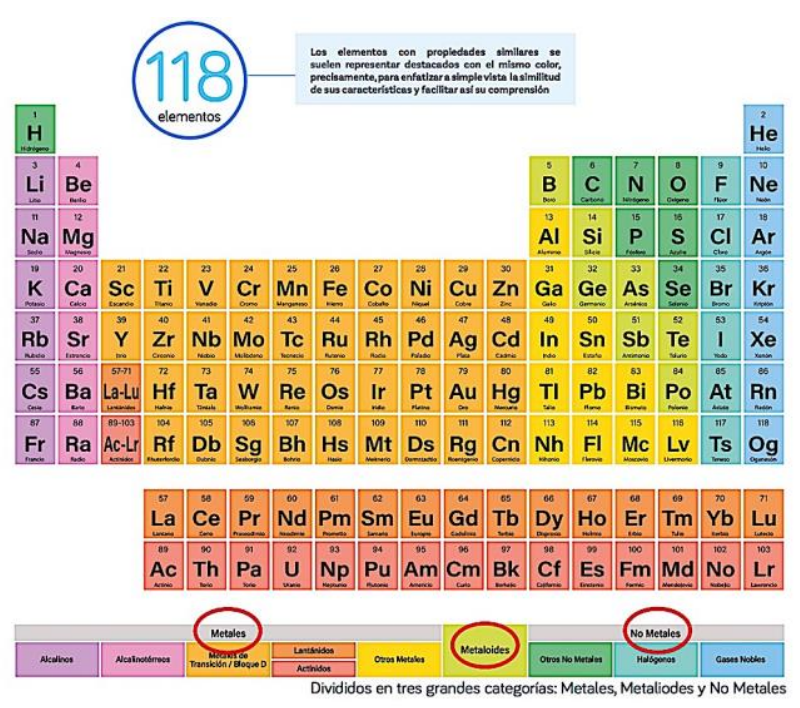

Figura 2 Tabla periódica de los elementos químicos Fuente: Química y Sociedad, 2019

\section{Metodología empleada}

Uno de los pasos más importantes y decisivos de la investigación es la elección del método o camino que llevará a obtener de la investigación resultados validos que respondan a los objetivos inicialmente planteados.

De esta decisión dependerá la forma de trabajo, la adquisición de la información, los análisis que se practiquen y por consiguiente el tipo de resultados que se obtengan. La selección del proceso de investigación guía todo el proceso investigativo y con base en el, se logra el objetivo de toda investigación (Ruiz, Borboa y Rodríguez, 2013).

\section{a) Enfoque de investigación}

Para lograr tener una aplicación de los elementos químicos mediante un dispositivo móvil, la presente investigación se realizó bajo un enfoque mixto. Hernández, Fernández y Baptista (2010) en su trabajo titulado Metodología de la Investigación, sostienen que todo trabajo de investigación se sustenta en dos enfoques principales: el enfoque cuantitativo y el enfoque cualitativo, los cuales de manera conjunta forman un tercer enfoque: El enfoque mixto. 
Este modelo mixto representa el más alto grado de integración o combinación entre los enfoques cualitativo y cuantitativo. Ambos se entremezclan o combinan en todo el proceso de investigación, o al menos, en la mayoría de sus etapas. Requiere de un manejo completo de los dos enfoques y una mentalidad abierta. Agrega complejidad al diseño de estudio, pero contempla todas las ventajas de cada uno de los enfoques (Hernández, Fernández y Baptista, 2010).

Se utilizó este enfoque de investigación mixto, ya que se recolectó, analizó y se vincularon datos cuantitativos y cualitativos, para responder al planteamiento de una problemática del entorno, lo que permitió conocer la necesidad de la población objetivo para entender como aprenden los alumnos tradicionalmente, los elementos químicos de la tabla periódica, así como también conocer la forma, en que les gustaría aprender mediante una aplicación tecnológica.

\section{b) Universo de estudio}

El universo de estudio de esta investigación está dirigido a los alumnos que cursan la asignatura de Química, en el Colegio de Bachilleres de Tabasco (COBATAB), como muestra se tomo al plantel 6, del municipio de Cunduacán, Tabasco, en la República Mexicana, que cursan el segundo semestre, ya que ellos harán uso de la aplicación como apoyo en su proceso de aprendizaje.

\section{c) Herramientas para la recolección de la información}

Para fundamentar la investigación se utilizaron tres herramientas de recolección de datos, los cuales fueron: el cuestionario, la observación y la entrevista.

La observación se usó porque en esta herramienta el investigador toma contacto directo, con el hecho o fenómeno social relacionado al problema motivo de estudio, logrando así tener una mejor cercanía ante la situación estudiada tal como ésta se da naturalmente, esta herramienta se empleó tanto en alumnos como en profesores.

En el caso del cuestionario, éste se utilizó para conocer las necesidades e intereses de los alumnos en lo que se refiere a la tabla periódica y el dispositivo móvil.
Así mismo, también se usó como herramienta de recolección de datos la entrevista con la experta que imparte la asignatura para escuchar de viva voz, los problemas que ha detectado de los alumnos cuando se les explica cada una de las parte de los elementos químicos.

\section{d) Modelo para el ciclo de vida y para el diseño de contenido del Software}

Para esta investigación se eligió el modelo lineal secuencial el cual sugiere un enfoque que comienza en un nivel de sistemas y progresa con el análisis, diseño, codificación, pruebas y mantenimiento (Pressman, 2010).

Para conducir los contenidos educativos se utilizó, el modelo de diseño instruccional llamado ADDIE ya que es un proceso sistemático, orientado a entornos virtuales, representado como un flujo de procesos que progresa de izquierda a derecha (Steven, 2017).

\section{Uso del modelo instruccional ADDIE}

Para el desarrollo del sitio Web se utilizó el modelo instruccional ADDIE, el cual es sencillo de usar y sirve para contemplar con detenimiento las fases de cualquier tipo de actividad de formación o diseño de material.

Etapa: Análisis

Se determinó el perfil de los alumnos

Se determinó las necesidades de la población objetivo

Se definió los contenidos apropiados Etapa: Diseño

Se establecieron los objetivos

Se estableció la estrategia Instruccional a utilizar Se estableció las especificaciones de la herramienta Se estableció un cronograma para trabajar Etapa: Desarrollo

Se trabajó con los desarrolladores de la herramienta

Se desarrollaron los contenidos educativos

Se desarrollaron los ejercicios prácticos

Se crear el ambiente virtual para el aprendizaje Etapa: Implementación

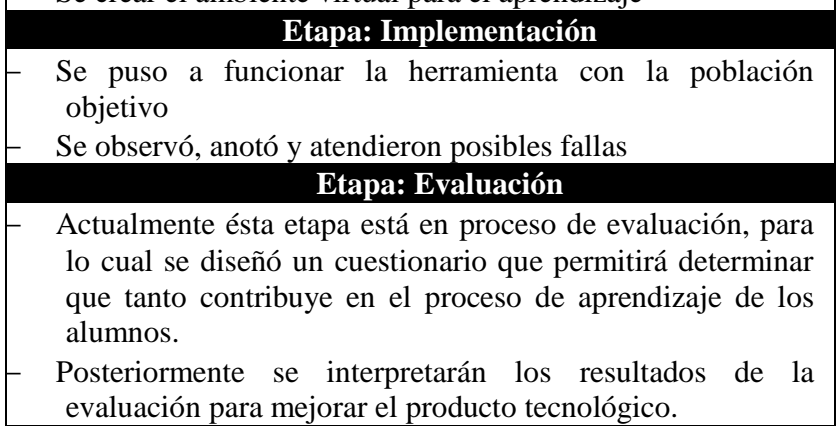

Tabla 1 Uso del modelos ADDIE

Fuente: Elaboración Propia 
Las fases del modelo ADDIE son 5 y que son un acrónimo de los términos Análisis, Diseño, Desarrollo, Implementación y Evaluación

Estos pasos pueden seguirse secuencialmente, o pueden ser utilizados de manera ascendente y simultánea a la vez.

En la Tabla 1, se presentan de manera simplificada las tareas realizadas por haber aplicado éste modelo instruccional ADDIE.

\section{Desarrollo de la herramienta}

Para llevar a cabo la elaboración de esta herramienta móvil fue necesario utilizar Adobe Photoshop CS4, para la edición de imágenes y Flash profesional CS4 para la creación de animaciones.

Adobe Photoshop CS4 se utilizó para trabajar y editar toda las imágenes, y fue de gran ayuda para modificar, retocar, deformar, rotar, crear efectos, etc.

También es un programa que nos permite realizar ciertas aplicaciones graficas, como Illustrator, pero su función principal es la de trabajar con imágenes el cual fue de gran utilidad para la aplicación móvil.

En la figura 3 se muestra la edición del logotipo de inicio de la aplicación.

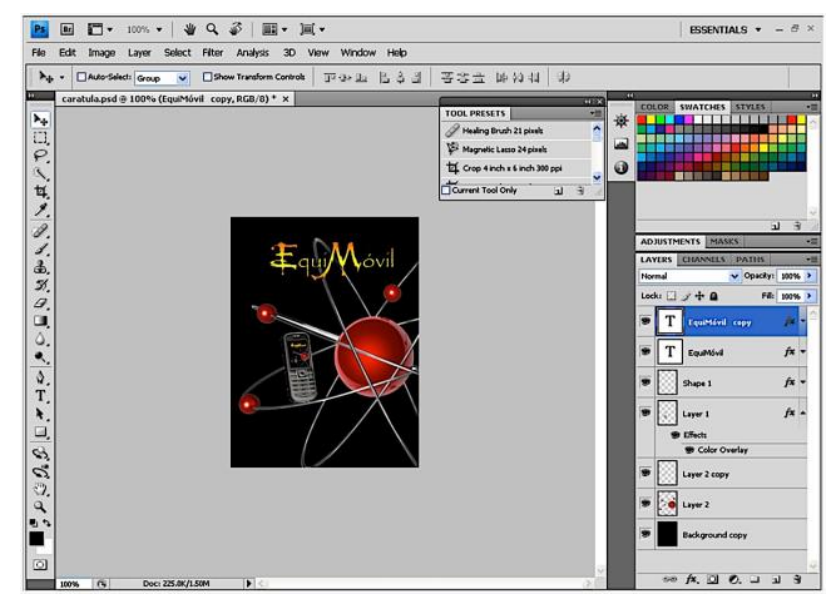

Figura 3 Pantalla de edición Adobe Photoshop CS4 Fuente: Elaboración Propia

Adobe Flash profesional CS4 es una aplicación de creación y manipulación de gráficos vectoriales con posibilidades de manejo de código mediante el lenguaje ActionScript en forma de estudio de animación que trabaja sobre fotogramas.
Este programa sirvió para la animación de botones, gráficos, y eventos que se utilizaron en el desarrollo de la aplicación (ver figura 4).

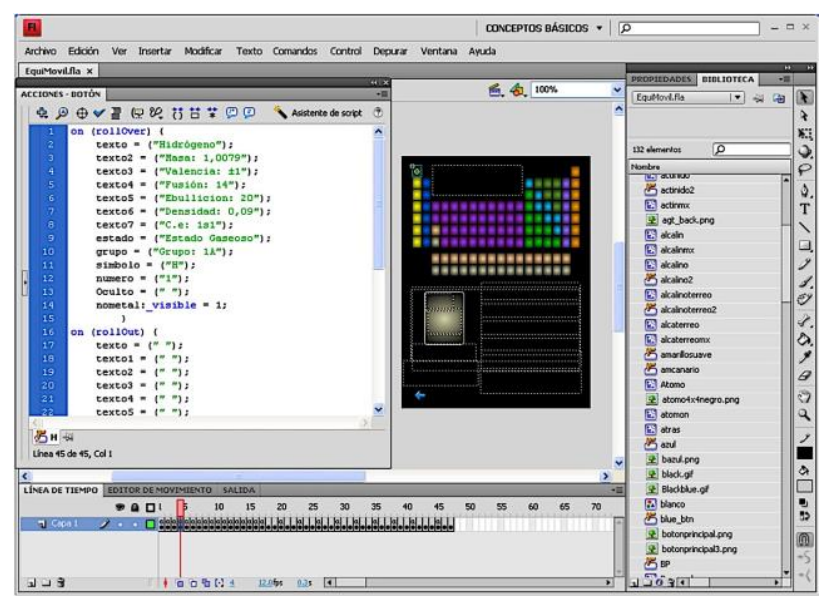

Figura 4 Usando Adobe Flash profesional CS4 Fuente: Elaboración Propia

\section{Presentando resultados}

Mediante un cuestionario que constaba de 9 preguntas, sirvió para conocer las características de la población objetivo, que se aplicó al grupo B del COBATAB No. 6 como población muestra. Se observó que el $86 \%$ de un total 43 alumnos mencionó poseer un celular (ver Gráfico 1).

\section{¿Posees un teléfono celular para tu uso?}

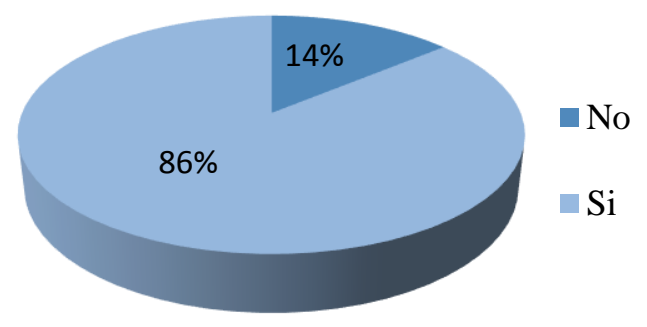

Gráfico 1 Porcentaje de alumnos que tienen celular Fuente: Elaboración Propia

Otra pregunta que se les hizo a los alumnos que tenían teléfono celular fue ¿Cual es la marca de tu teléfono celular?, en donde $43 \%$ menciono tener Sony mientras el $22 \%$ señaló poseer un celular Nokia, un $24 \%$ otro tipo de celular, el $8 \%$ Motorola y finalmente el $3 \%$ un LG (ver Gráfico 2). 
¿Cuál es la marca de tu teléfono?

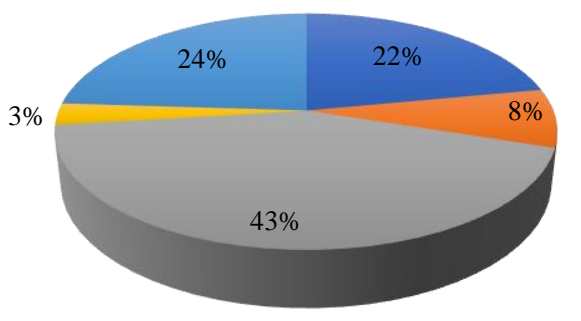

- Nokia

Motorola

Sony

$\square$ LG

- Otro tipo

Gráfico 2 Marcas de teléfonos celulares Fuente: Elaboración Propia

Por otra parte, el $65 \%$ de los alumnos que tiene un dispositivo móvil, manifestaron que les gustarían usar una aplicación que le sirva para aprender los elementos químicos de la tabla periódica en la asignatura de Química (ver Gráfico 3).

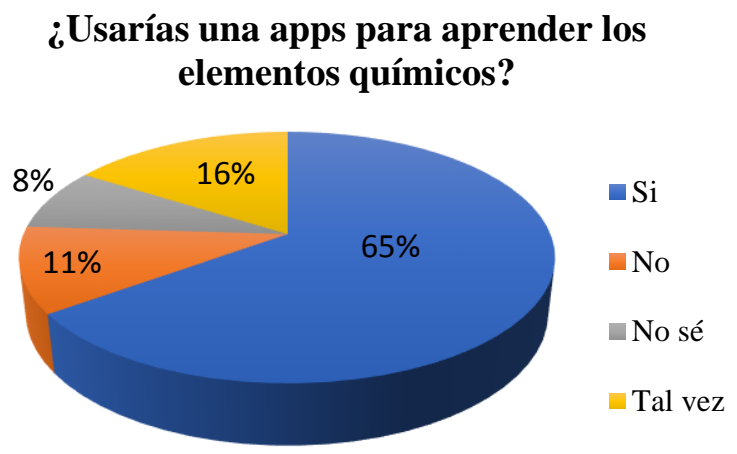

Gráfico 3 Alumnos que usarían la aplicación Fuente: Elaboración Propia

De igual forma, el $70 \%$ de los alumnos mencionaron que puede ser de utilidad la aplicación para su dispositivo móvil, que aborde este tema a como se muestra en el Gráfico 4.

¿Crees que sea de utilidad una apps para aprender los elementos químicos?

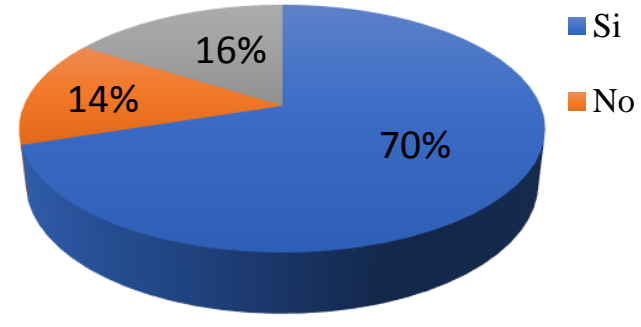

Gráfico 4 Alumnos que creen de utilidad la aplicación Fuente: Elaboración Propia

\section{La herramienta tecnológica móvil}

Como resultado de esta investigación, se obtuvo la herramienta tecnológica, cuyo contenido esta divida en 4 módulos (ver figura 5) que son: Tabla General; Tipo de elemento, que se muestra organizado en grupos; Búsqueda; y un Test, en el cual se presenta una serie de preguntas relacionada con la información presentada.

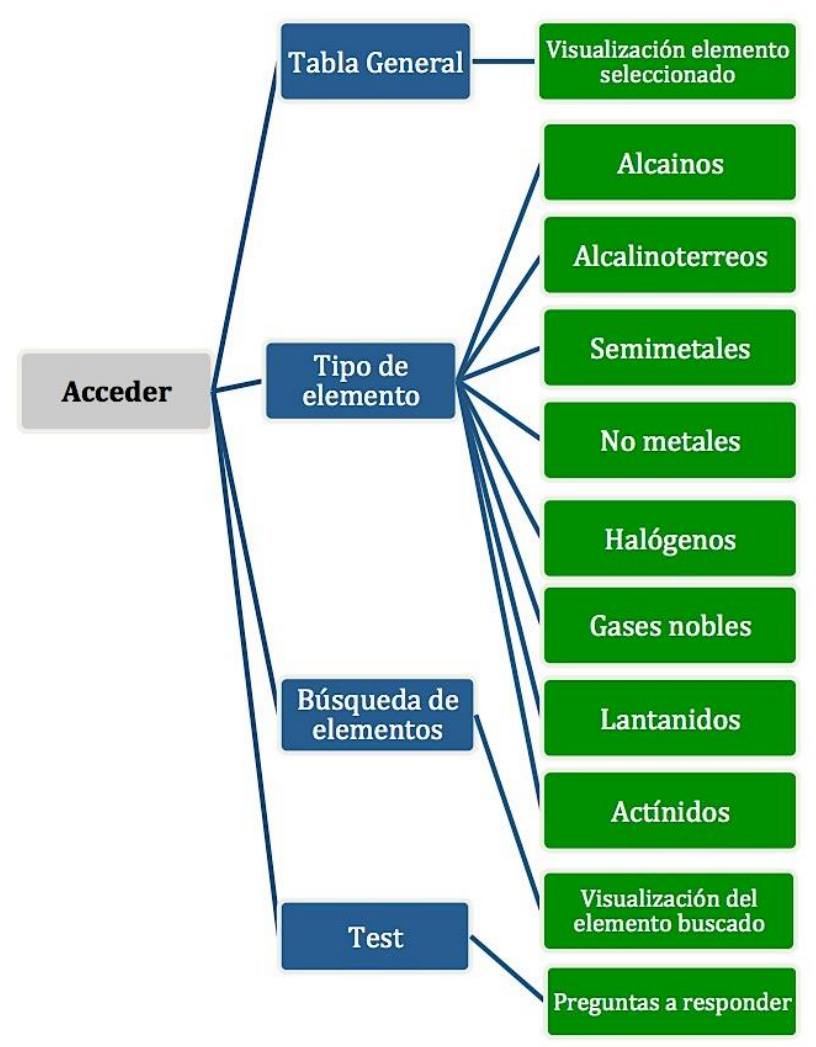

Figura 5 Diagrama de navegación de la aplicación Fuente: Elaboración Propia

La primer pantalla que ven los usuarios de la aplicación móvil muestra el menú principal con las opciones seleccionables, las cuales son Tabla general, Tipo de elemento, Búsqueda y Test (ver figura 6). 


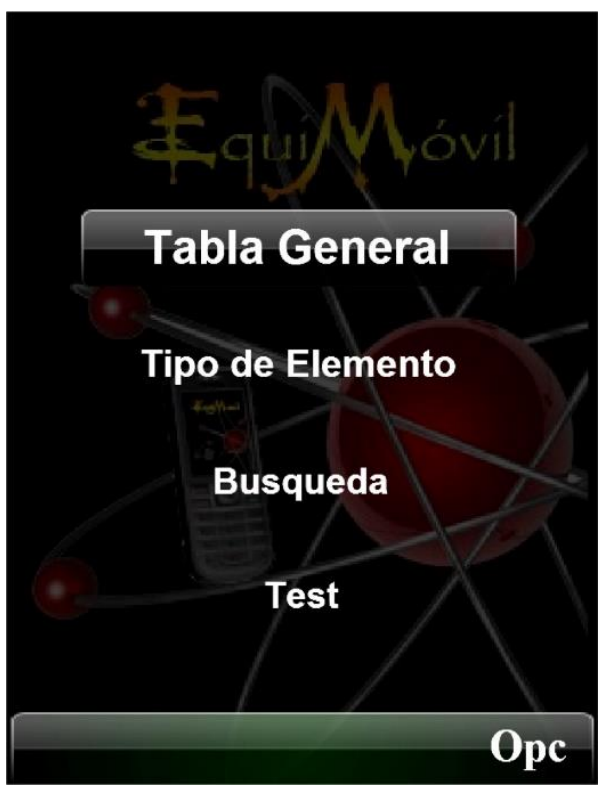

Figura 6 Opciones generales de la aplicación Fuente: Elaboración Propia

En el módulo de "Tabla General", muestra los elementos químicos de manera general, similar al de un formato impreso, donde los grupos están representados con colores particulares y dejando ver el símbolo de cada elemento en íconos pequeños, que al seleccionar, se maximiza y muestra la información a detalle. Esto está representado en la Figura 7.

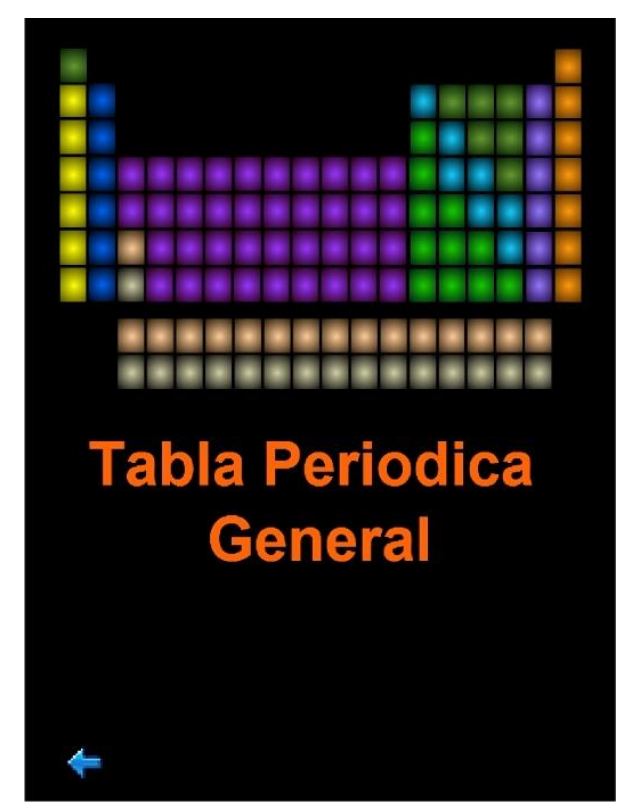

Figura 7 Pantalla de la Tabla Periódica General Fuente: Elaboración Propia

En éste mismo módulo de "Tabla General", si se selecciona alguno de los elementos representados con colores específicos, se presentarán los datos particulares del mismo, como son: la masa, valencia, fusión, densidad, entre otros (ver figura 8).



Figura 8 Pantalla Tabla Periódica General Fuente: Elaboración Propia

En el módulo de "Tipo de elemento" se muestra la estructura general de la tabla periódica similar al de un formato impreso, pero solo se presentan los elementos que pertenecen al un mismo grupo, resaltados con un color en particular, dejando ver la información a detalle de cada uno de ellos, al momento que son seleccionados. Esta función está representada en la Figura 9.

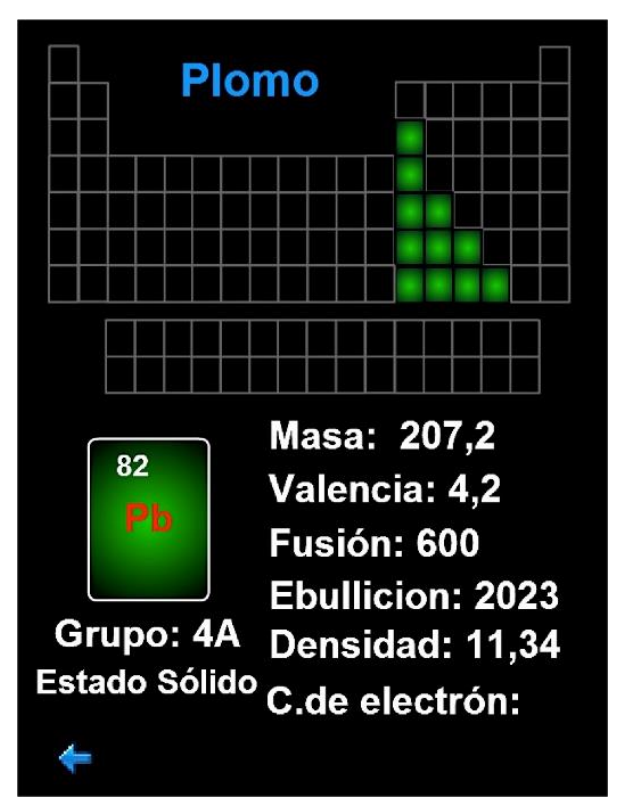

Figura 9 Tipos de elementos de la tabla periódica de un mismo grupo

Fuente: Elaboración Propia

Si le elige la opción de "Búsqueda", se presentará un cuadro donde se podrá escribir el nombre o el símbolo del elemento químico y si lo encuentra mostrará en pantalla sus datos generales del mismo (ver figura 10). 




Figura 10 Pantalla de la opción de "Búsqueda" Fuente: Elaboración Propia

En la Figura 11, se muestra la pantalla de preguntas de la opción de "Test", que permite que el usuario elija alguna de las 6 posibles respuestas que esta relacionada con los símbolos de elementos químicos, según el que se le pregunte en pantalla.

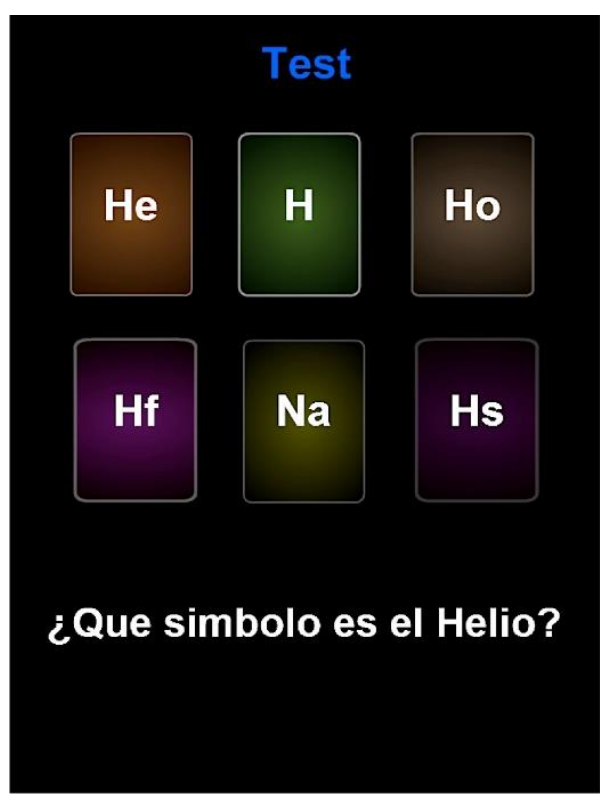

Figura 11 Pantalla de preguntas de "Test" Fuente: Elaboración Propia

Cabe hacer mención que actualmente la aplicación desarrollada en esta investigación, está en proceso de evaluación con alumnos del COBATAB plantel 6, del municipio de Cunduacán, Tabasco en México; para lo cual se diseñó un cuestionario que permitirá determinar que tanto contribuye en el proceso de aprendizaje de los mismos.

\section{Conclusiones}

La aplicación desarrollada en esta investigación permite que los usuarios tengan acceso a los datos contenidos en cada elemento químico de la tabla periódica. Para facilitar la utilización de la misma, ésta cuenta con un menú para acceder a los elementos de acuerdo al formato convencional o por medio de la agrupación por colores, el cual facilita visualizar los elementos que conforman a los distintos grupos por su característica.

Haber realizado una aplicación para teléfonos móviles donde su estructura está organizada y su interfaz es agradable, fácil de utilizar y manejable, además de ser gratuita, ayudará en el proceso de enseñanza-aprendizaje de los elementos químicos de la tabla periódica a los alumnos del Plantel 6 del Colegio de Bachilleres del municipio de Cunduacán, del Estado de Tabasco.

Es importante recalcar que la aplicación es totalmente portable y puede ser utilizada por cualquier usuario que posea un teléfono celular de la marca Sony y Nokia, ya que fueron los modelos que más se usan dentro del ámbito estudiantil del COBATAB No. 6, y es que en la actualidad, es más factible portar un dispositivo de este tipo que un equipo de cómputo. Esto se debe a que un teléfono celular es manejable y pequeño, en cambio una computadora, es más voluminosa y no todos tienen acceso a una.

\section{Referencias}

Esquivel, F. y Hernández, J. (2016). "Evolución del Mercado de Smartphones en México en 2015". Consultado el 10 de marzo de 2016. Dirección de internet: http://www.theciu.net/nwsltr/479_2Distro.html

Hernández, Sampieri Roberto, Fernández C. Baptista L. P. (2010); Metodología de la Investigación; Ed. Mc Graw Hill (4ta ed.). México, DF.

Instituto Nacional de Estadística, Geografía e Informática, INEGI (2018). Comunicado de prensa núm. 179/19. Consultado el 10 de mayo de 2019 de http://www.beta.inegi.org.mx/contenidos/salade prensa/boletines/2019/OtrTemEcon/ENDUTIH _2018.pdf 
Martínez Peniche, J. R.(2015). La Tabla Periódica (Los Elementos y la Estructura Atómica). Consultado el 27 de octubre de 2018 de http://cea.quimicae.unam.mx/Estru/tabla /01_Introduccion.htm

PoderPDA, (2012). Estadisticas Mundiales de Smartphones y Celulares 2012. Consultado el 22 de Marzo de 2017 de http://www.poderpda.com/investigacion-y desarrollo/estadisticas-mundiales-desmartphones-y-celulares-2012/

Pressman, R. (2010). Ingenieria de Software, un enfoque practico. Ed. McGraw-Hill Interamericana, MEXICO

Química y Sociedad (2019). El ABC de la TABLA PERIÓDICA. Consultado por Internet el 22 de Marzo de 2019 de https://www.quimicaysociedad.org/pdf/DossierABC-Tabla-Periodica.pdf

Rolando Alamilla, R., Esquivel, F. (2015). Ecosistema Competitivo del Mercado de Smartphones 4T14". Consultado el 12 de mayo de 2015 de http://www.theciu.net/nwsltr/350Distro.html

Ruiz Medina, M. I. ; Borboa Quintero, M.del S; Rodríguez Valdez, J.C. (2013). El enfoque mixto de investigación en los estudios fiscales. Consultado el 3 de septiembre de 2017 de http://www.eumed.net/rev/tlatemoani/13/estudi os-fiscales.pdf

Soler Pellicer, Y. (2009), Consideraciones sobre la tecnología educativa en el proceso de enseñanza-aprendizaje. Una experiencia en la asignatura Estructura de Datos, Revista Iberoamericana de Educación ISSN: 16815653, No. 49/2, Consultado el 30 de octubre de 2017 ,

http://www.rieoei.org/expe/2863Soler.pdf

Steven, J.(2017); Instructional Systems, College of Education, Penn State University. McGriff. Consultado el 12 de junio de 2018 de http://disenoinstrucional.files.wordpress.com/20 07/09/addiemodel.doc 